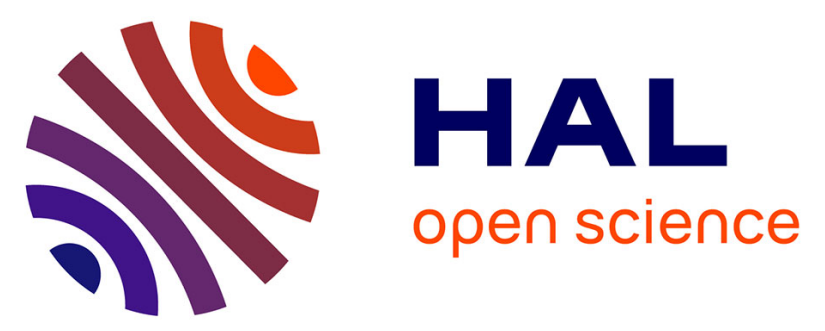

\title{
Towards Quantitative Void Fraction Measurement With an Eddy Current Flowmeter for Fourth Generation Sodium Cooled Fast Reactors: A Simplified Model
}

Mithlesh Kumar, Philippe Tordjeman, Wladimir Bergez, Matthieu Cavaro, Kevin Paumel, Jean-Philippe Jeannot

\section{To cite this version:}

Mithlesh Kumar, Philippe Tordjeman, Wladimir Bergez, Matthieu Cavaro, Kevin Paumel, et al.. Towards Quantitative Void Fraction Measurement With an Eddy Current Flowmeter for Fourth Generation Sodium Cooled Fast Reactors: A Simplified Model. IEEE Transactions on Nuclear Science, 2016, vol. 63 ( $\mathrm{n}^{\circ} 3$ ), pp. 1-6. 10.1109/TNS.2016.2542191 . hal-01561622

\author{
HAL Id: hal-01561622 \\ https://hal.science/hal-01561622
}

Submitted on 13 Jul 2017

HAL is a multi-disciplinary open access archive for the deposit and dissemination of scientific research documents, whether they are published or not. The documents may come from teaching and research institutions in France or abroad, or from public or private research centers.
L'archive ouverte pluridisciplinaire HAL, est destinée au dépôt et à la diffusion de documents scientifiques de niveau recherche, publiés ou non, émanant des établissements d'enseignement et de recherche français ou étrangers, des laboratoires publics ou privés. 


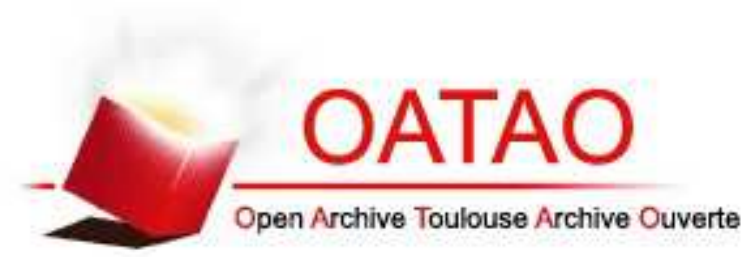

\section{Open Archive TOULOUSE Archive Ouverte (OATAO)}

OATAO is an open access repository that collects the work of Toulouse researchers and makes it freely available over the web where possible.

This is an author-deposited version published in : http://oatao.univ-toulouse.fr/ Eprints ID : 18059

To link to this article : DOI: $10.1109 /$ TNS.2016.2542191

URL : http://dx.doi.org/10.1109/TNS.2016.2542191

To cite this version : Kumar, Mithlesh and Tordjeman, Philippe and Bergez, Wladimir and Cavaro, Matthieu and Paumel, Kevin and Jeannot, Jean-Philippe Towards Quantitative Void Fraction Measurement With an Eddy Current Flowmeter for Fourth Generation Sodium Cooled Fast Reactors: A Simplified Model. (2016) IEEE Transactions on Nuclear Science, vol. 63 (n 3). pp. 16. ISSN 0018-9499

Any correspondence concerning this service should be sent to the repository administrator: staff-oatao@ listes-diff.inp-toulouse.fr 


\title{
Towards Quantitative Void Fraction Measurement With an Eddy Current Flowmeter for Fourth Generation Sodium Cooled Fast Reactors: A Simplified Model
}

\author{
M. Kumar, Ph. Tordjeman, W. Bergez, M. Cavaro, K. Paumel, and J. P. Jeannot
}

\begin{abstract}
We propose an experimental methodology for the purpose of quantitative void fraction measurements in fourth generation Sodium cooled fast reactors with a standard Eddy Current Flow Meter (ECFM) sensor. The methodology consists of using the technique of ellipse fit and correlate the fluctuations in the angle of inclination of this ellipse with the void fraction. This methodology is applied in this paper to an ideal configuration of periodic grooves on solid aluminium cylinder with various volumic fractions. The effects of physical parameters such as coil excitation frequency, coil current and motion have been studied. The first results show that ECFM is sensitive to void fractions between $0.3 \%$ and $6.9 \%$. It further demonstrates that the response to void fraction is insensitive to the mean velocity of the two-phase medium.
\end{abstract}

Index Terms-COMSOL, eddy current flow meter, ellipse fitting, sodium cooled fast reactors, void fraction.

\section{INTRODUCTION}

$\mathbf{S}$ ODIUM cooled fast reactor is under R\&D as one of the potential candidates for generation IV nuclear reactors. Besides being more efficient and economical, generation IV nuclear reactors are expected to possess enhanced safety features. The presence of gas bubbles in a Sodium cooled Fast Reactor (SFR) is an important safety issue from the point of view of nuclear reactor operation. For example, in primary loop of an SFR, it can lead to reactivity changes in the core, reduce the heat transfer between sub-assemblies and the liquid metal and cause damage to the pumps. Here, monitoring and control of gas bubbles becomes a necessity. The incidental presence of gas bubbles in secondary sodium loop can serve as an indicator of a leak in the sodium gas heat exchanger.

Manuscript received June 30, 2015; revised January 04, 2016 and February 03, 2016; accepted March 09, 2016. Date of publication May 30, 2016; date of current version June 21, 2016. This work was supported by the CEA, DEN, Nuclear Technology Department, Saint-Paul-lez-Durance, France.

M. Kumar is with the Institut de Mécanique des Fluides de Toulouse, 31400 Toulouse, France, and also with the CEA, DEN, Nuclear Technology Department, F-13108 Saint-Paul-lez-Durance, France (e-mail: mithlesh.kumar@imft.fr; mithlesh.kumar@cea.fr).

P. Tordjeman and W. Bergez are with the Institut de Mécanique des Fluides de Toulouse, 31400 Toulouse, France.

M. Cavaro, K. Paumel, and J. P. Jeannot are with the CEA, DEN, Nuclear Technology Department, F-13108 Saint-Paul-lez-Durance, France.

Color versions of one or more of the figures in this paper are available online at http://ieeexplore.ieee.org.

Digital Object Identifier 10.1109/TNS.2016.2542191
The measurement of void fraction in liquid sodium is a difficult problem because it is opaque, aggressive, at high temperature and mostly inaccessible. The simultaneous measurement of sodium velocity and void fraction is also a challenging issue because these two physical quantities are interdependent. Therefore many conventional void fraction measurement techniques are no more usable in these situations. Existing void fraction detection and measurement techniques for liquid metal flows have their own advantages and limitations. For example, low frequency acoustic techniques are limited by the maximum frequency condition, which consequently puts an upper limit on the size of the bubbles and maximum detectable void fraction.

Eddy current type flowmeter (ECFM) were initially proposed for flow rate measurement and the detection of flow blockage in the sub-assemblies of SFR [1]-[4]. ECFM is non intrusive, can be operated remotely, compact in size so that it can be used in narrow channels, and gives large a.c. signals [5]-[8]. Furthermore, it could be optimized to minimize temperature induced errors [9], [10]. With all these advantages, ECFM makes an attractive potential candidate for void fraction measurements in liquid metal two phase flows.

An ECFM usually consists of one primary coil $(P)$ and two secondary coils $\left(S_{1}\right.$ and $S_{2}$ ) placed coaxially on either sides of the primary coil, with electrically conducting medium in motion through the core of the coils. An external AC current, $I=I_{0} \cos \omega t$ in $P$ sets up a magnetic flux in the conducting medium. This magnetic flux is sensed by $S_{1}$ and $S_{2}$ as emf $V_{1}$ and $V_{2}$, respectively. In a single phase flow, the difference voltage, $\Delta V=V_{2}-V_{1}$ is a linear function of the flow rate at low magnetic Reynolds number $R e_{m}=\sigma \mu_{0} U \delta$, where $\sigma$ is the electrical conductivity of the medium, $\mu_{0}$ is the magnetic permeability of vacuum, $U$ is the characteristic flow velocity, and $\delta$ is the penetration depth of the magnetic field in the medium $\left(\delta=\sqrt{2 / \sigma \mu_{0} \omega}\right)$. A typical value of $R e_{m}$ for ECFM in an SFR is $R e_{m} \approx 0.1$, [9].

For two-phase flow, $V_{1}$ and $V_{2}$ are modified due to the effects of the distribution of the dispersed phase. Nakamoto et al. [11] carried out phase sensitive synchronous detection at core exit in JOYO experimental fast reactor in 1984 using an ECFM in probe type configuration. They demonstrated that the device output voltage is proportional to void fraction from $0.1 \%$ up to $2 \%$. The results showed that an ECFM is 40 times more sensitive to void than the conventional permanent magnet 


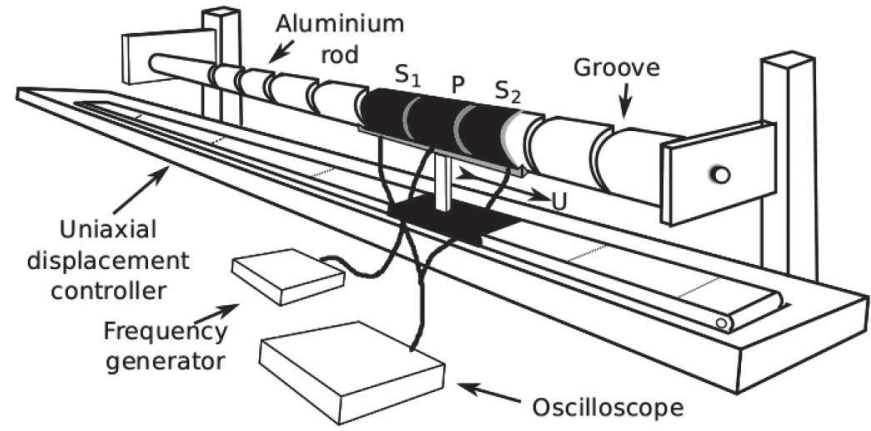

Fig. 1. Experimental set-up with ECFM assembly $\left(S_{1}, P, S_{2}\right)$.

TABLE I

SPECIFICATIONS OF THE RODS USED IN EXPERIMENTS

\begin{tabular}{llllll}
\hline$\alpha(\%)$ & $D(\mathrm{~mm})$ & $d_{g}(\mathrm{~mm})$ & $d_{1}(\mathrm{~mm})$ & $d_{2}(\mathrm{~mm})$ & $N_{g}$ \\
\hline 6.9 & 38.5 & 4.23 & 13.85 & 3 & 18 \\
0.3 & 38.5 & 0.375 & 16.6 & 1.4 & 17 \\
0 & 38.5 & - & - & - & - \\
$1 \mathrm{gr}$ & 38.5 & 4.23 & - & 3 & 1 \\
\hline
\end{tabular}

flowmeters. In the present study, we explore further the properties and capabilities of this device. We use the technique of ellipse fit [12], [13] for analysis of the induced signals $V_{1}$ and $V_{2}$, and demonstrate that ECFM is sensitive to large void fractions and the void signal is decoupled from the velocity signal. This shows that an ECFM can be used simultaneously as a flowmeter and void detector in plug flow type.

\section{MATERiAls AND Methods}

\section{A. Experiments: Set Up and Method}

A moving ECFM assembly was designed (Fig. 1). It consists of three coils ( $P, S_{1}$ and $S_{2}$ ) of diameter $40 \mathrm{~mm}$ each and length $30 \mathrm{~mm}$ for $P$ and $20 \mathrm{~mm}$ for $S_{1}$ and $S_{1}$, with copper winding of 70 turns for $P$ and 50 turns for $S_{1}$ and $S_{2}$. ECFM is fixed on a Uniaxial displacement controller, which can move in the range of velocity, $U=0.001 \mathrm{~m} / \mathrm{s}$ to $1 \mathrm{~m} / \mathrm{s}$. The conducting fluid is modeled by an aluminium rod along which the ECFM moves, which is a configuration similar to a plug flow. To simulate void, grooves were machined at the rod surface. Four aluminium rods were used in the experiments (Table I): a rod with large grooves with void volume fraction, $\alpha=6.9 \%$, rod with small grooves, $\alpha=0.3 \%$, plain rod with no grooves, $\alpha=0 \%$ and a rod with only one groove, 1 gr. $\alpha$ is the ratio of the total groove volume to the volume of the plain rod. In Table I, D is the diameter of the rod, $d_{g}$ is depth of groove, $d_{1}$ is the separation between two grooves, $d_{2}$ is the length of a groove, and $N_{g}$ is the total number of grooves. All dimensions are in $\mathrm{mm}$. The experiments were realized at room temperature for which the electrical conductivity of the aluminium rod is $\sigma=3.8 \quad 10^{7} \mathrm{~S} / \mathrm{m}$. We use a frequency generator to excite the primary coil at $500 \mathrm{~Hz}(\delta \approx$ $3.7 \mathrm{~mm})$ and $1000 \mathrm{~Hz}(\delta \approx 2.6 \mathrm{~mm})$. The current intensity for all the experiments is $250 \mathrm{~mA}$. The voltage induced in $S_{1}$ and $S_{2}$ is recorded in an oscilloscope as a Lissajous curve. Ellipse fit on this Lissajous curve gives us the tilt angle $\beta$. The advantage of this system is that $\alpha(\%)$ can be controlled precisely and

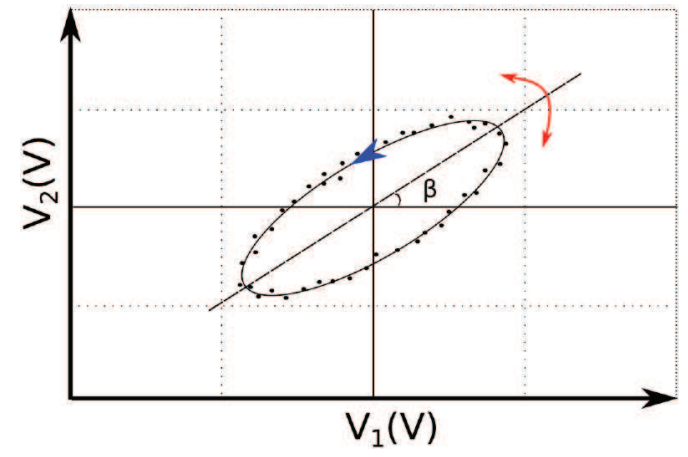

Fig. 2. Typical Lissajous curve of signals $V_{1}(t)$ and $V_{2}(t)$.

is exactly known contrary to a system with liquid metal loop. Experiments with this system are highly reproducible because the system is deterministic.

Ellipse fitting algorithms are popular in the fields of digital signal processing and image analysis [12], [13]. When two signals $V_{1}(t)$ and $V_{2}(t)$ are out of phase and satisfy certain conditions, their Lissajous curve is an ellipse (Fig. 2). $V_{1}(t)$ and $V_{2}(t)$ are functions of the impedance of the electrically conducting medium under study. In two-phase liquid metalgas flows, the impedance of the two-phase medium depends on the distribution and volume fraction of the dispersed phase. This allows one to use ellipse fitting as a tool to analyze the impedance of the medium under investigation. In this way, various ellipse parameters could be used to characterize the two phase flows.

If the induced voltages in the two secondary coils $S_{1}$ and $S_{2}$ are, $V_{1}=\left|V_{1}\right| \sin \left(\omega t-\theta_{1}\right)$ and $V_{2}=\left|V_{2}\right| \sin \left(\omega t-\theta_{2}\right)$ respectively, then the tilt angle of the fitted ellipse is given by,

$$
\beta=\left|\frac{1}{2} \arctan \left(\frac{-2 \cos (\Delta \theta)\left|V_{1}\right|\left|V_{2}\right|}{\left|V_{1}\right|^{2}-\left|V_{2}\right|^{2}}\right)\right|
$$

where, $\Delta \theta=\theta_{1}-\theta_{2} . \Delta \theta$ is the extra phase introduced by the void in one coil with respect to the other. Physically, it is governed by perturbations of Faraday and motion induced eddy currents which have different phases. At low magnetic Reynolds number $R e_{m}, \Delta \theta$ is linear with $U$, but very close to $0^{\circ}$ or $180^{\circ}$, [14], [15]. The numerical simulation described in the next section suggests that for $0 \leq R e_{m} \leq 0.12$, $0 \leq \Delta \theta(\mathrm{rad}) \leq 0.025$ and consequently $0.9997 \leq \cos \Delta \theta \leq$ 1. Therefore, in the absence of voids the ellipse approaches a straight line inclined near $45^{\circ}$. When a void is under $S_{1},\left|V_{1}\right|$ becomes much larger than $\left|V_{2}\right|$ and $V_{1}(t)$ has a slightly different phase than $V_{2}(t)$. We get a prominent tilted ellipse. The effects of void passage on phase and amplitude of $V(t)$ can be found in this reference [16]. The tilt fluctuates about $45^{\circ}$ when voids pass through the flow cross-section. We can relate the fluctuation in the tilt angle to characterize the void presence. From the time signal of $\beta$ we calculate the amplitude of oscillation $\Delta \beta$ by FFT algorithm.

\section{B. Numerical Simulations}

A Finite Element Simulation of the problem was realized using COMSOL simulation software with Magnetic Fields 


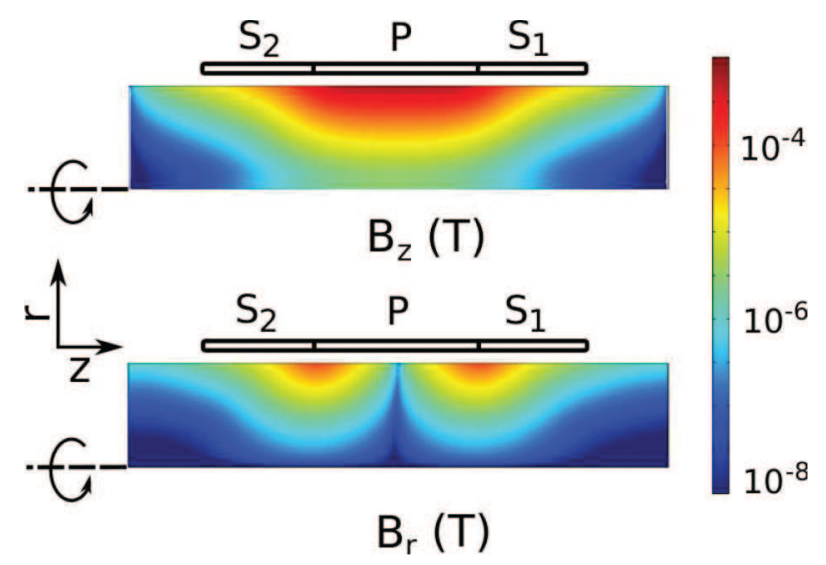

Fig. 3. Distribution of axial and radial magnetic flux density inside a plain conducting rod. $(I=250 \mathrm{~mA}, \nu=1000 \mathrm{~Hz}, U=0 \mathrm{~m} / \mathrm{s})$.

physics in Frequency domain. The equation solved is the advection - diffusion of $\mathbf{A}$, the magnetic vector potential:

$$
\nabla^{2} \mathbf{A}-\mu \sigma(\mathbf{U} \times(\nabla \times \mathbf{A}))+j \omega \sigma \mathbf{A}=\mu_{0} \mathbf{J}^{e} .
$$

$\mathbf{J}^{e}$ represents the externally applied current density and it is non-zero only in the domain which represents the primary coil. Since the grooves do not break the path of eddy currents and the externally applied current density $\mathbf{J}^{e}$ is azimuthal, the problem is $2 \mathrm{D}$ axisymmetric and we need to solve for only one component of the magnetic vector potential:

$$
\mathbf{A}=A(r, z) \hat{\mathbf{e}}_{\theta} .
$$

Magnetic insulation condition is used at the edges of the computational domain. It consists in prescribing all the components of magnetic vector potential as zero on these edges. Grid size independence was verified by using three different mesh size settings: finer, extra fine and extremely fine respectively. Minimum mesh size varies at edges, boundaries and interfaces (up to $10^{-2} \mathrm{~mm}$ ).

Magnetic fields distribution in a plain conducting rod, when $P$ coil is excited by $I=250 \mathrm{~mA}$ at $\nu=1000 \mathrm{~Hz}$ is of the order of $0.1 \mathrm{mT}$ and is shown in Fig. 3. Notice that the axial magnetic flux density, $B_{z}$ is uniformly distributed in $z$-direction within the $P$ coil. The radial magnetic flux density, $B_{r}$ is positive at interface between $P$ and $S_{1}$ coil while it is negative at interface between $P$ and $S_{2}$ coil. This induces opposite flow induced eddy currents at $S_{1}$ and $S_{2}$ through $\sigma(\vec{U} \times \vec{B})$ term in Eqn. (2). It increases voltage $V_{1}$ in $S_{1}$ while decreases voltage $V_{2}$ in $S_{2}$ by the same amount. $\Delta V=V_{1}-V_{2}$, is thus proportional to $U$.

\section{RESUlTS AND Discussions}

Expected linearity of the amplitude of $\Delta V(\mathrm{~V})$ with $U(\mathrm{~m} / \mathrm{s})$ is verified in Fig. 4 for the plain rod. Measured sensitivity is around $0.07 \mathrm{mV} / \mathrm{ms}^{-1}$. The linear fitting shows a finite value equal to $0.074 \mathrm{mV}$ at $U=0 \mathrm{~m} / \mathrm{s}$, which corresponds to unbalanced secondary coils. This is also the order of magnitude of the voltages induced in individual secondary coils. Notice that the contribution of flow induced term is one order smaller even

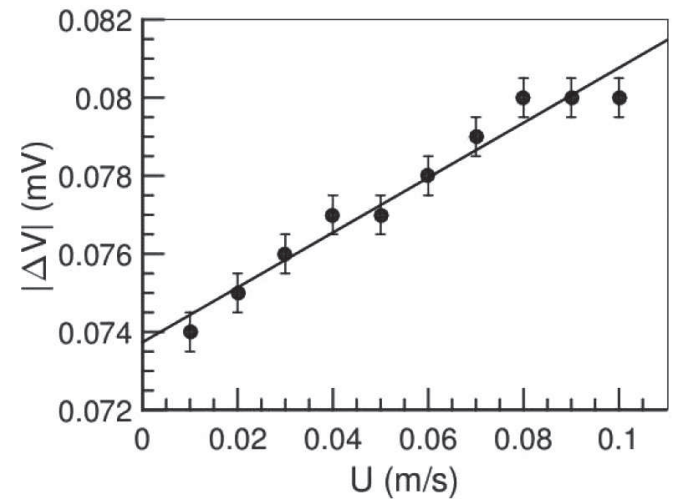

Fig. 4. Amplitude of $\Delta V(\mathrm{~V})$ vs $U(\mathrm{~m} / \mathrm{s})(\alpha=0 \%$ and $\nu=1000 \mathrm{~Hz})$. Experiment: $(\bullet)$, Linear fit $(-)$.

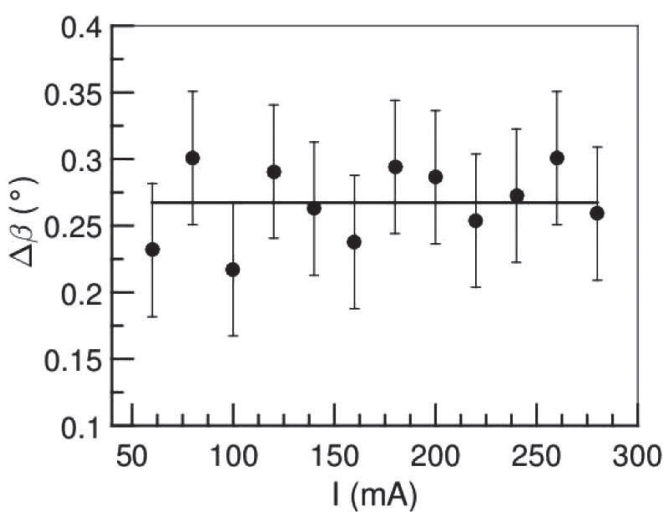

Fig. 5. $\Delta \beta\left(^{\circ}\right)$ vs $I(\mathrm{~mA}), U=0.1 \mathrm{~m} / \mathrm{s}, \nu=1000 \mathrm{~Hz}, \alpha=6.9 \%$.

at highest $U: 0.007 / 0.074 \sim 0.1$. This is because $R e_{m} \sim 0.1$ in our experiments.

Since the secondary signal voltages $V_{1}$ and $V_{2}$ are linear function of the primary coil excitation current, $I$, it is to be expected from Eqn. (1) that $\beta$ signal is independent of $I$. This is confirmed by Fig. 5 which shows that the change in primary coil excitation current, $I$ has no effect on the amplitude of the $\beta$ signal within the range of experimental uncertainty.

Fig. 6-9 show the response of the ECFM signal to the presence of grooves. In all these cases, primary coil $P$ is excited by current $I=250 \mathrm{~mA}$ at frequency $\nu=1000 \mathrm{~Hz}$. The numerical results have been rescaled by factor 0.6 and a small static $\beta$ was removed from experimental data in Figs. 6-9 for comparison with the numerical results. For the same control parameters $(I, U, \nu)$, the only difference between experiments and numerical simulation is the symmetry of ECFM assembly. While in simulations we have a perfectly symmetric system, in practice we cannot manufacture a perfectly balanced ECFM assembly which is also evident from Fig. 4. In the case of perfectly symmetric system, the intercept at $U=0 \mathrm{~m} / \mathrm{s}$ is expected to be $0 \mathrm{~V}$ but Fig. 4 shows a value $0.074 \mathrm{mV}$. Fig. 10 shows the effect of asymmetry in ECFM assembly on the $\beta$ signal amplitude, $\Delta \beta$. Keeping the number of turns in each secondary coil $S_{1}$ and $S_{2}$ as constant, their lengths were altered as $L_{s}-k L_{s}$ and $L_{s}+k L_{s}$ respectively in the numerical simulations. In such a case, factor of asymmetry, $k$ can refer to compactness of winding in secondary coils. We observe that $\Delta \beta$ is maximum for 


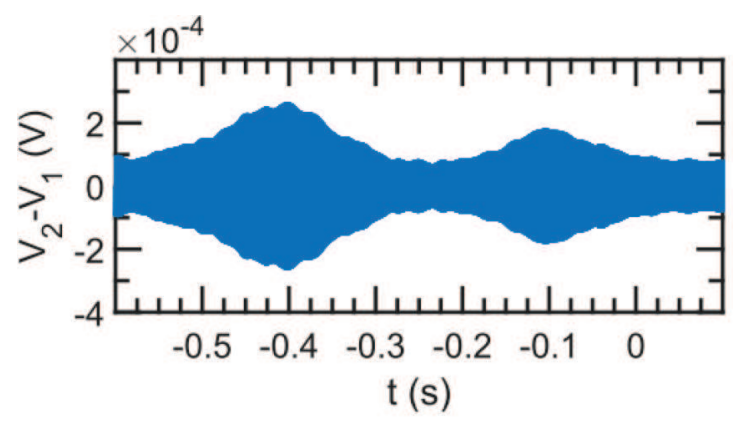

(a)

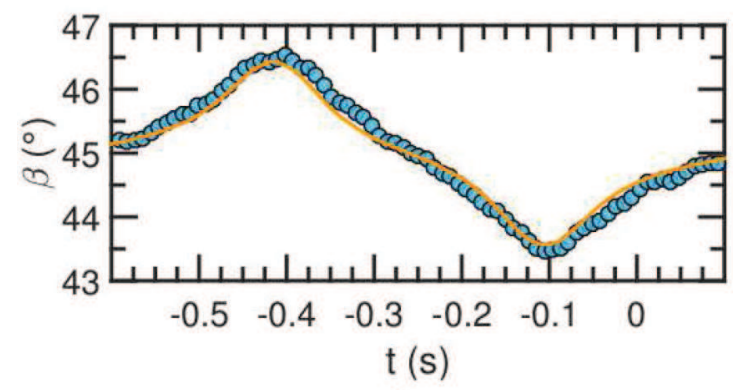

(b)

Fig. 6. Rod with one groove $U=0.1 \mathrm{~m} / \mathrm{s}$ and $\nu=1000 \mathrm{~Hz}$. (a) $\Delta V$ vs time. (b) $\beta$ vs $t(s)$. (: experimental data; : numerical simulation).

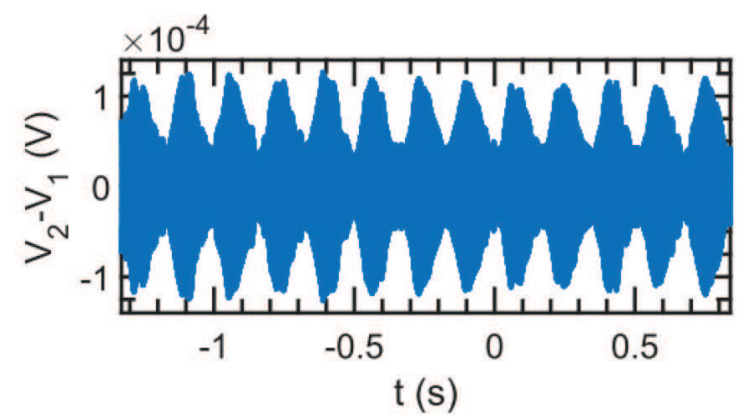

(a)

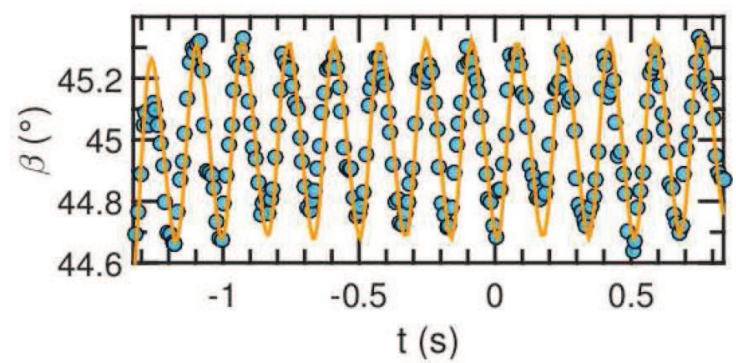

(b)

Fig. 7. $\alpha=6.9 \%, U=0.1 \mathrm{~m} / \mathrm{s}$ and $\nu=1000 \mathrm{~Hz}$. (a) $\Delta V$ vs time. (b) $\beta$ vs $t(s)$. (: experimental data; : numerical simulation).

a perfectly symmetrical system $(k=0)$ while it decreases as a function of asymmetry. In practice, ECFM assembly is never perfectly symmetric. The ratio of $\Delta \beta$ obtained from numerical simulation and experiments at same parameter values give us an idea about the degree of asymmetry in the experimental ECFM assembly. There are many reasons for asymmetry besides compactness. The profile of $\Delta \beta$ with asymmetry should be expected

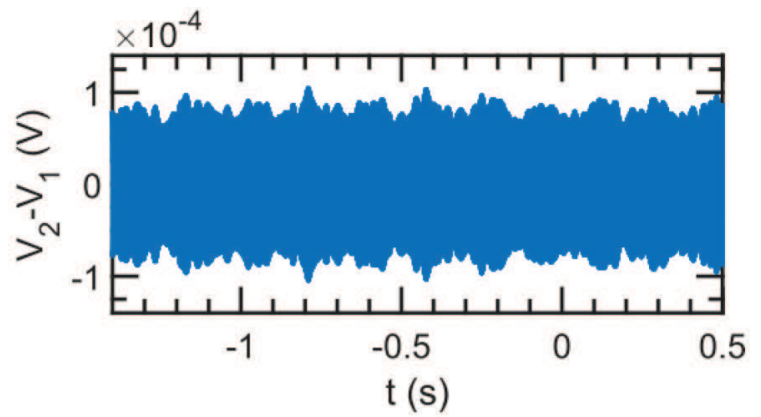

(a)

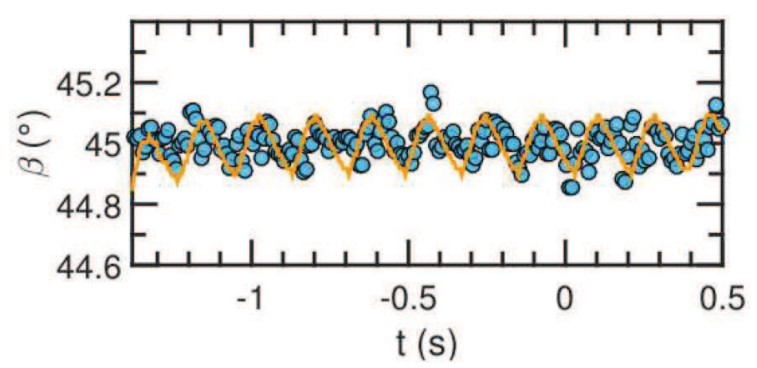

(b)

Fig. 8. $\alpha=0.3 \%, U=0.1 \mathrm{~m} / \mathrm{s}$ and $\nu=1000 \mathrm{~Hz}$. (a) $\Delta V$ vs time. (b) $\beta$ vs $t(s)$. (: experimental data; : numerical simulation).

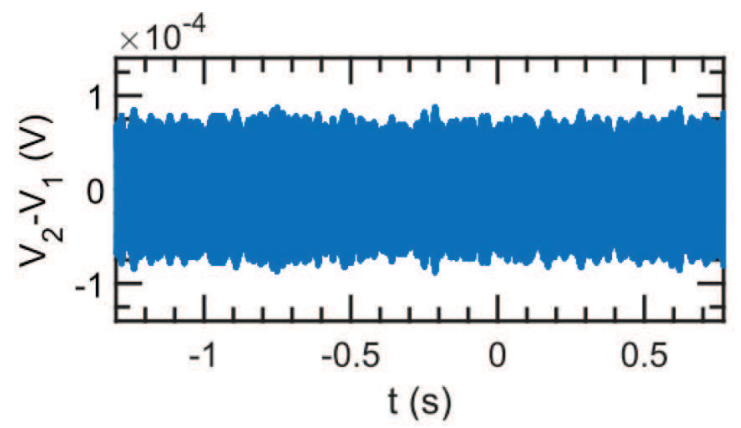

(a)

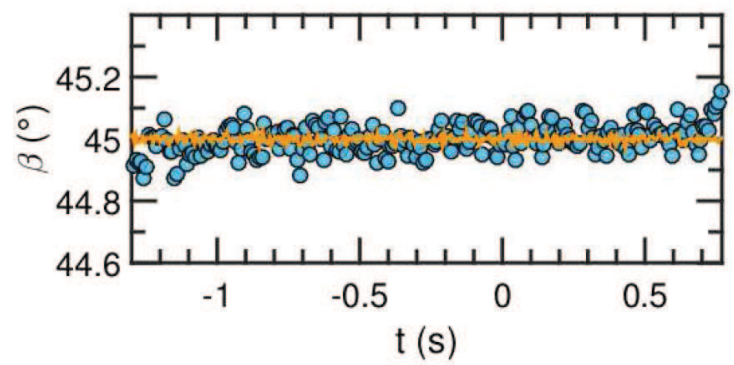

(b)

Fig. 9. $\alpha=0 \%, U=0.1 \mathrm{~m} / \mathrm{s}$ and $\nu=1000 \mathrm{~Hz}$. (a) $\Delta V$ vs time. (b) $\beta$ vs $t(s)$. (: experimental data; : numerical simulation).

to vary according to the type of asymmetry (for example: compactness, lift off, distance from primary coil etc.). It is advisable to calibrate the device for this factor after fabrication.

In Fig. 6 we see the signal obtained for a one groove rod. We observe the perturbation in both $\Delta V$ as ECFM assembly translates over the groove at $U=0.1 \mathrm{~m} / \mathrm{s}$, and its corresponding signature on the tilt angle signal, $\beta(\mathrm{t})$. We notice that $\beta(\mathrm{t})$ without groove is close to $45^{\circ}$ and that the variation due to 


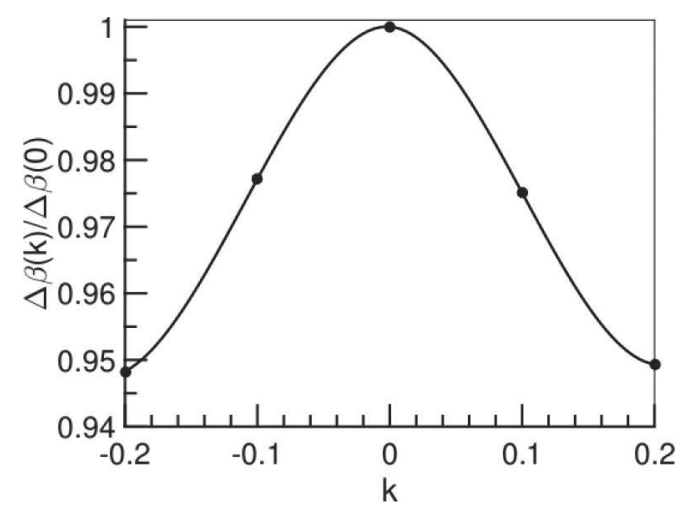

Fig. 10. $\Delta \beta(k)$ is maximum for a perfectly symmetric system $(k=0)$ and it decreases as a function of asymmetry $(k \neq 0)$. COMSOL simulation for $\alpha=$ $6.9 \%$ at $U=0.01 \mathrm{~m} / \mathrm{s}, I=200 \mathrm{~mA}$ and $\nu=1000 \mathrm{~Hz}$.

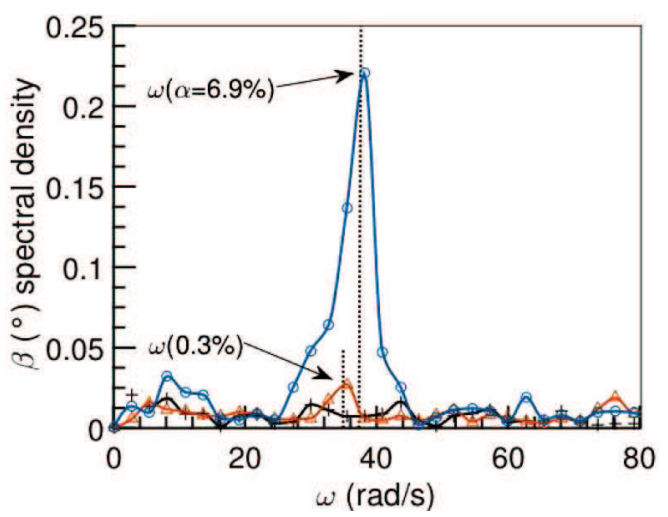

Fig. 11. $\beta$ spectral density for + , and for $\alpha=0.0,0.3$ and $6.9 \%$ respectively at $U=0.1 \mathrm{~m} / \mathrm{s}$ and $\nu=1000 \mathrm{~Hz}$.

the grooves has a maximum amplitude of $1.5^{\circ}$ which is easily detectable. The first peak corresponds to the passage of the groove inside $S_{1}$ and second peak to that inside $S_{2}$. The distance between the two peaks is $0.3 \mathrm{~s}$, which at $U=0.1 \mathrm{~m} / \mathrm{s}$ gives a length scale, $L=3 \mathrm{~cm}$ which is the separation between the two secondary coils. Besides that, parameter $\beta$ seems more clean as compared to the difference signal.

The effect of void fraction on $\beta$ signal can be observed by comparing Fig. 7-9. The standard deviations for the three $\beta(\mathrm{t})$ curves are 0.044 for $0 \%, 0.062$ for $0.3 \%$ and 0.276 for $6.9 \%$. It shows that at $0.3 \%$ we are close to the limit of detection. However, FFT gives the amplitude of oscillation of $\beta$ signal, $\Delta \beta$ with a reasonable accuracy (Fig. 11). Furthermore, peak to peak distance is equal to the spatial frequency of grooves. This gives information on distribution of the dispersed phase through Fourier analysis.

Fig. 6-9 also demonstrate an excellent agreement between the experimental results and the numerical simulations for $\beta$ signal.

Fig. 12 shows the effect of frequency on $\Delta \beta$. When the frequency increases, $\Delta \beta$ tends towards saturation. This happens because at low frequencies the magnetic field can penetrate upto the core of the rod. As we increase the frequency, the magnetic flux is excluded from the core leading to an exponential decrease in the skin depth.

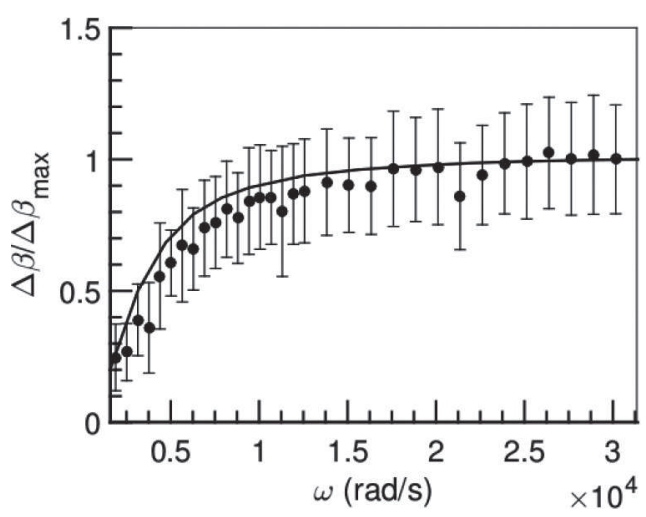

Fig. 12. Normalized amplitude $\Delta \beta$ as a function of frequency for $\alpha=$ $6.9 \%, U=0.1 \mathrm{~m} / \mathrm{s}$ and $I=200 \mathrm{~mA}$. (•: experimental data; —: numerical simulation; $\left.\Delta \beta_{\max }=\Delta \beta(\nu=4800 \mathrm{~Hz})\right)$.

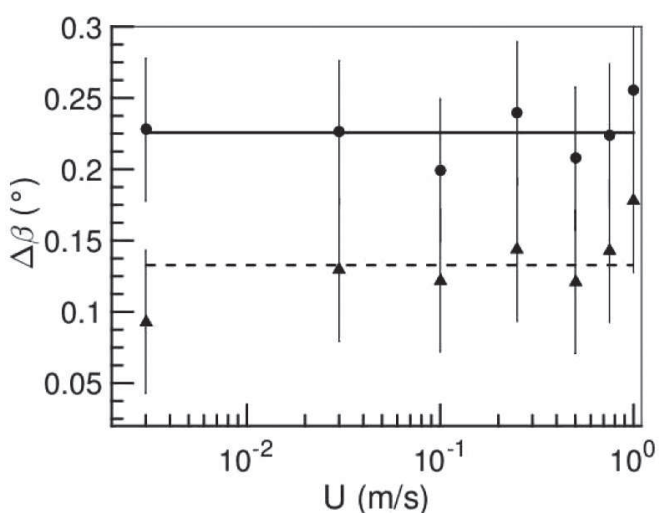

Fig. 13. $\Delta \beta$ vs $U$ for $\alpha=6.9 \%, I=200 \mathrm{~mA}, \nu=1000 \mathrm{~Hz}(\bullet)$ and $500 \mathrm{~Hz}$ $(\boldsymbol{\Delta})$.

We have shown before that the contribution of flow induced potential is one order smaller than average induced potentials in each secondary coils. Theoretically, $\beta(U)$ for $R e_{m} \leq 0.12$ is, $\beta(U) \leq|1 / 2 \arctan (-20)| \Rightarrow \Delta \beta(U)=$ $\beta(0)-\beta(1) \leq 45.0^{\circ}-43.5^{\circ}=1.5^{\circ}$. Flow fluctuations of the order of $1 \mathrm{~m} / \mathrm{s}$ should change $\Delta \beta$ up to a maximum $1.5^{\circ}$. But in our experiments, there are no flow fluctuations and therefore $\Delta \beta$ should be independent of $U$. This is confirmed in Fig. 13, where $\Delta \beta$ is independent of the velocity. This result suggests that in steady flows, $\Delta \beta$ depends only on $\alpha$, however the period of $\beta$ oscillations depends on the velocity. Moreover, this implies that the effect of void fraction is mainly through Faraday induction rather than the Lorentz force.

The effect of homogenization defined by mean groove separation length $\lambda=0.5\left(d_{1}+d_{2}\right)$ has not been studied. The change in $\lambda$ affects the groove frequency in FFT spectrum of $\beta(t) . \Delta \beta=0$ for continuous gas. It is expected that $\Delta \beta$ will start to decrease when the axial length of the annular slugs approach the length of the primary coil in dimension. This will typically happen around $\alpha \geq 30 \%$, [17].

\section{CONCLUSIONS}

An experimental method has been introduced to study potentiality of a 3-coil flow-through type ECFM to measure void 
fraction for liquid metal two-phase flows. Periodic grooves on solid aluminium cylinder was used as a model of liquid metal two-phase flow. The model experimental set up used in this paper allows us to control well the experimental parameters such as void volume, location, velocity etc. We use the technique of ellipse fit and correlate the fluctuations in tilt of this ellipse with the void fraction. The same problem was also modeled numerically in COMSOL simulation software. The results show a good agreement between the experiments and the numerical simulations. The main results are: $\beta$ signal for the single groove case demonstrate the detection capability of ECFM for large grooves. By comparing the $\beta$ signal for various rods, we find that it is sensitive to $\alpha . \Delta \beta \propto \alpha^{n} n \approx 0.7$, is the trend of variation of $\Delta \beta$ with the void fraction. signal for $\alpha=0.3 \%$ is noisy. This is approximately the lower limit of void fraction that can reliably be detected using this method. $\Delta \beta$ increases with frequency and reaches an asymptotic value. This is related to its dependence on penetration depth of the magnetic fields in the medium. $\Delta \beta$ is independent of the primary coil excitation current. The experiments conducted at various velocity values show that for the normal reactor conditions ( $U \sim 1 \mathrm{~m} / \mathrm{s}$ ), $\Delta \beta$ does not change with $U$ assuming a steady flow. This is important for reliable measurements of void fraction. In this study we find that ECFM signals analyzed with the technique of ellipse fit is promising. Compared to acoustic methods, the upper limit on detectable $\alpha$ is much larger. The signals are not only fast and large but are also decoupled from the mean flow velocity. Further studies are needed to confirm these results for a general liquid metal two-phase flow in an SFR [17]. In particular, the results presented here do not account for possible randomness in $\alpha$ distribution and velocity fluctuations.

\section{ACKNOWLEDGEMENT}

The authors would like to acknowledge the contributions of Hervé Ayroles from Signaux et Images team and Grégory Ehses from Interface team at IMFT Toulouse for their technical assistance.

\section{REFERENCES}

[1] H. Lehde and W. Lang, AC electromagnetic induction flow meter, U.S., Patent 2435 043. 1948, vol. 2.

[2] D. E. Wiegand, "Summary of an analysis of the eddy-current flowmeter," IEEE Trans. Nucl. Sci., NS-15. no. 1, pp. 28-36, 1968.

[3] A. Dohi, M. Oda, and S. Iida, "Development of an in-core flow meter for the LMFBR," Proc. Int. Conf. Liquid Technology in Energy Production, 1976.

[4] M. Hirayama, "Theoretical model of an eddy-current flowsensor," IEEE Trans. Nucl. Sci., NS-24, no. 5, pp. 2021-2030, 1977.

[5] J. A. Shercliff, The Theory of Electromagnetic Flow-Measurement, , 1962, CUP Archive.

[6] K. Ara, "A differential transformer with temperature and excitationindependent output," IEEE Trans. Instrum. Meas., vol. 21, no. 3, pp. 249$255,1972$.

[7] C. C. Feng, W. E. Deeds, and C. V. Dodd, "Analysis of eddy-current flowmeters," J. Appl. Phys., vol. 46, no. 7, pp. 2935-2940, 1975.

[8] R. C. Baker, "Electromagnetic flowmeters for fast reactors," Progr. Nucl. Energy, vol. 1, no. 1, pp. 41-61, 1977.

[9] P. Sharma, S. Suresh Kumar, B. Nashine, R. Veerasamy, B. Krishnakumar, P. Kalyanasundaram, and G. Vaidyanathan, "Development, computer simulation and performance testing in sodium of an eddy current flowmeter," Ann. Nucl. Energy, vol. 37, no. 3, pp. 332-338, 2010.

[10] S. Sureshkumar, M. Sabih, S. Narmadha, N. Ravichandran, R. Dhanasekharan, C. Meikandamurthy, G. Padmakumar, R. Vijayashree, V. Prakash, and K. Rajan, "Utilization of eddy current flow meter for sodium flow measurement in FBRs," Nucl. Eng. Des., vol. 265, pp. 1223-1231, 2013.

[11] K. Nakamoto, S. Tamura, K. Ishii, H. Kuwahara, N. Ohyama, and T. Muramatsu, "Application of an eddy-current type flowmeter to void detection at the lmfbr core exit," Nucl. Eng. Des., vol. 82, no. 2, pp. 393-404, 1984.

[12] P. M. Ramos, F. M. Janeiro, M. Tlemçani, and A. C. Serra, "Recent developments on impedance measurements with dsp-based ellipse-fitting algorithms," IEEE Trans. Instrum. Meas., vol. 58, no. 5, pp. 1680-1689, 2009.

[13] A. Fitzgibbon, M. Pilu, and R. B. Fisher, "Direct least square fitting of ellipses," IEEE Trans. Pattern Anal. Mach. Intell., vol. 21, no. 5, pp. 476480, 1999.

[14] D. Buchenau, S. Eckert, G. Gerbeth, R. Stieglitz, and M. Dierckx, "Measurement technique developments for lbe flows," J. Nucl. Mater., vol. 415, no. 3, pp. 396-403, 2011.

[15] J. Priede, D. Buchenau, and G. Gerbeth, "Contactless electromagnetic phase-shift flowmeter for liquid metals," Meas. Sci. Technol., vol. 22, no. 5, pp. 055402, 2011.

[16] M. Kumar, P. Tordjeman, W. Bergez, and M. Cavaro, "Note: Void effects on eddy current distortion in two-phase liquid metal," Rev. Sci. Instrum., vol. 86, no. 10, pp. 106104, 2015.

[17] J. E. Cha, Y. C. Ahn, K. W. Seo, H. Y. Nam, J. H. Choi, and M. H. Kim, "The performance of electromagnetic flowmeters in a liquid metal twophase flow," J. Nucl. Sci. Technol., vol. 40, no. 10, pp. 744753, 2003. 